\title{
Quantitative trait loci that modulate trabecular bone's risk of failure during unloading and reloading
}

\author{
Engin Ozcivici ${ }^{\mathrm{a}, *}$, Weidong Zhang ${ }^{\mathrm{b}}$, Leah Rae Donahue ${ }^{\mathrm{b}}$, Stefan Judex ${ }^{\mathrm{c}}$ \\ a Department of Mechanical Engineering, Izmir Institute of Technology, Urla, Izmir 35430, Turkey \\ b The Jackson Laboratory, Bar Harbor, ME 04609, USA \\ c Department of Biomedical Engineering, Stony Brook University, Stony Brook, NY 11794, USA
}

\section{A R T I C L E I N F O}

Article history:

Received 8 October 2013

Revised 1 February 2014

Accepted 22 March 2014

Available online 31 March 2014

Edited by: Robert Recker

\section{Keywords:}

Mechanical Loading

Stress

Recovery

Genetic research

Finite element method

QTL

\begin{abstract}
A B S T R A C T
Genetic makeup of an individual is a strong determinant of the morphologic and mechanical properties of bone. Here, in an effort to identify quantitative trait loci (QTLs) for changes in the simulated mechanical parameters of trabecular bone during altered mechanical demand, we subjected 352 second generation female adult (16 weeks old) BALBxC3H mice to 3 weeks of hindlimb unloading followed by 3 weeks of reambulation. Longitudinal in vivo microcomputed tomography $(\mu \mathrm{CT})$ scans tracked trabecular changes in the distal femur. Tomographies were directly translated into finite element (FE) models and subjected to a uniaxial compression test. Apparent trabecular stiffness and components of the Von Mises (VM) stress distributions were computed for the distal metaphysis and associated with QTLs. At baseline, five QTLs explained $20 \%$ of the variation in trabecular peak stresses across the mouse population. During unloading, three QTLs accounted for $14 \%$ of the variability in peak stresses. During reambulation, one QTL accounted for $5 \%$ of the variability in peak stresses. QTLs were also identified for mechanically induced changes in stiffness, median stress values and skewness of stress distributions. There was little overlap between QTLs identified for baseline and QTLs for longitudinal changes in mechanical properties, suggesting that distinct genes may be responsible for the mechanical response of trabecular bone. Unloading related QTLs were also different from reambulation related QTLs. Further, QTLs identified here for mechanical properties differed from previously identified QTLs for trabecular morphology, perhaps revealing novel gene targets for reducing fracture risk in individuals exposed to unloading and for maximizing the recovery of trabecular bone's mechanical properties during reambulation.
\end{abstract}

(c) 2014 Elsevier Inc. All rights reserved.

\section{Introduction}

A principal function of bone is to withstand mechanical loads acting upon it. Through its capacity for dynamic remodeling, bone tissue processes external physical signals as informative cues to adapt its mass, structure and mechanical properties [1,2]. Mechanical loads are required for bone maintenance and growth while unloading causes erosion of bone morphology and strength [3-5]. Inherently, the relation between loading and specific bone variables is regulated at the genetic level. Interestingly, bone does not necessarily perceive and process similar mechanical cues in the same manner across individuals with different genotypes, giving rise to substantially different molecular and morphologic outcomes [6-8]. The identity of the genetic locations regulating bone's response to (un)loading, while largely unknown, would provide targets towards protecting individuals from bone loss during deprivation of mechanical loads and maximizing bone gain during the application of loads.

\footnotetext{
* Corresponding author at: Department of Mechanical Engineering, Rm 110, Izmir Institute of Technology, Urla, Izmir 35430, Turkey. Fax: +90 2327506701

E-mail address: enginozcivici@iyte.edu.tr (E. Ozcivici).
}

In the absence of differences in the mechanical loading environment, many studies have investigated quantitative trait loci (QTLs) and the associated genetic polymorphisms that explain variations in trabecular and cortical BMD and bone architecture across individuals during homeostasis [9-15]. Because bone's structural mechanical properties are more closely associated with fracture risk than BMD or bone architecture $[16,17]$, these efforts were expanded to determine genetic locations that modulate bone's mechanical properties including failure load, stiffness, or yield strength. Because of the greater complexity determining bone's mechanical properties rather than BMD or morphology, relatively little is known about mechanical genotype/phenotype relations. Genomic regions for bone's mechanical properties differ from QTLs identified for BMD [9,18-22], not surprisingly because BMD as measured by DXA is only one of many factors that determine strength [23].

QTL studies focusing on BMD or morphology of trabecular bone are less common than those targeting cortical bone [10,12]. QTL studies are often performed in mice, taking advantage of readily available genetic manipulations for follow-up studies [24], but the assessment of murine trabecular morphology requires high-resolution imaging methodologies such as micro computed tomography $(\mu \mathrm{CT})$. Further, due to its sub millimeter size and open porous structure, mechanical testing of 
trabecular compartments of mouse bones is difficult to achieve. Not surprisingly, little is known about the genetic variations that determine trabecular bone's mechanical properties. Even less is known about those QTLs that modulate changes in trabecular bone's mechanical properties due to altered mechanical demand.

As an alternative to invasive mechanical testing of trabecular bone, mechanical properties can be assessed in silico by using finite element (FE) models that are constructed from the structural information of $(\mu) C T$ images [25]. Simulated tests using FE models not only corroborate well with experimentally measured mechanical properties of bone [26-28] but can also provide detailed spatial information of regions within the trabecular structures that are at greater risk of failure. Further, since FE models can be derived directly from non-invasive in vivo scans, longitudinal changes of mechanical properties can be readily computed.

We recently subjected a large heterogeneous mouse population to unloading and reambulation and contrasted QTL for baseline trabecular morphology with those associated with changes in trabecular morphology and tissue mineral density for the two (un)loading protocols [29]. Trabecular bone's simulated mechanical properties, and changes in them during altered levels of weightbearing, are presented in detail in our companion article [65]. Here, we sought to identify QTLs for outcome variables that determine bone's mechanical integrity prior to and during unloading and reambulation. Because of dissociations between trabecular morphology and mechanical properties when gravitational loading is removed and reintroduced (as shown in the companion article), we hypothesized that QTLs for trabecular bone mechanics will overlap with QTLs for trabecular morphology at baseline and that this overlap will diminish for unloading and reambulation.

\section{Methods}

\section{Experimental design}

The Institutional Animal Care and Use Committee of Stony Brook University reviewed and approved all procedures. The experimental protocol has been described previously in a study that established the role of genetics on trabecular morphology during un- and reloading [29]. Briefly, female adult ( 16 weeks old, $n=466$ ) mice of F2 generation from female BALB/CByJ (BALB, high response to unloading) and male $\mathrm{C} 3 \mathrm{H} / \mathrm{HeJ}(\mathrm{C} 3 \mathrm{H}$, low response to unloading) inbred mice were selected. Mice were exposed to 3 weeks of hindlimb unloading followed by 3 weeks of unconstrained reambulation [30,31]. In vivo $\mu$ CT scans were administered at baseline $(n=466)$, after unloading $(n=359)$, and after reambulation $(\mathrm{n}=352)$. Sample sizes were not identical at the three $\mu \mathrm{CT}$ scan time points primarily because of scheduling conflicts for the time consuming in vivo scans. Mice that could not be scanned were sacrificed. Throughout the protocol, all mice were single-housed and had ad libitum access to food and water during a 12 hour light/ dark cycle. Upon the last $\mu \mathrm{CT}$ scan (22 weeks old), mice were sacrificed and the spleen was extracted and stored in $-80{ }^{\circ} \mathrm{C}$ for DNA extraction and analysis.

\section{Skeletal phenotyes by in vivo $\mu C T$}

With mice under isoflurane, the left and right metaphyses of the distal femur were $\mu \mathrm{CT}$ scanned (vivaCT 40 , Scanco Medical, Switzerland) at a voxel size of $17.5 \mu \mathrm{m}$ as described in more detail in the companion article [65]. The selected voxel size was small enough to exceed the minimal recommended voxel size for assessing bone's microstructure in rodents with $\mu \mathrm{CT}$ [32]. Grey scale $\mu \mathrm{CT}$ images were subjected to Gaussian blurring and thresholding and trabecular bone was separated from surrounding cortical bone using a semi-automatic algorithm [33]. Each $\mu \mathrm{CT}$ scan delivered an estimated CT dose of $340 \mathrm{mGy}$ to the anatomical region that was scanned. This dose did not elicit changes in the trabecular architecture of control and hindlimb unloaded mice in this study [29].

\section{Finite element modeling}

The finite element (FE) model that evaluated mechanical parameters of trabecular bone is discussed in our companion article [65]. Briefly, $\mu \mathrm{CT}$ voxels used to describe the morphologic variables [29] were directly converted into $17.5 \mu \mathrm{m}$ mechanical elements in the FE model. A frictionless test compressed the ROI under a $1 \mathrm{~N}$ uniformly distributed load [34]. Standard material properties were assigned to each mechanical element [35-37]. During post-processing, only voxels pertaining to trabecular bone were analyzed. Apparent stiffness values were calculated as the ratio between prescribed loads and displacements. Von-Mises (VM) stresses (also called equivalent stress), a mechanical variable that transforms all stress components into a single scalar variable, were computed for elements within the trabecular structure. Peak VM stress (PVM), median VM stress (MVM) and skewness of the VM stress distribution (SVM) were reported.

\section{Genotyping and QTL mapping}

Tail tips from F2 female mice were submitted to the SNP Genotyping Service at The Jackson Laboratory. For this study, 89 SNPs known to be polymorphic between the BALB and $\mathrm{C} 3 \mathrm{H}$ inbred strains were selected for genotyping. QTL analysis was performed for each phenotype using the statistical software R/QTL (version 1.16-6) [38]. For genome-wide one-dimensional scans, pseudo markers were generated at 2-cM spacing for each chromosome and scans were performed using 256 imputations [39]. Statistical thresholds, equivalent to $\alpha$-values, of $1 \%, 5 \%, 10 \%$, and $63 \%$ were calculated from one thousand permutations [40]. QTL with LOD scores above the $1 \%$ threshold were considered strong QTL, while those above 63\% were considered suggestive QTL [41]. As an example of determining the $5 \%$ threshold, we assume that No QTL is present in the genome, generate LOD scores randomly with 1000 permutations, and then select the 95th\%. If our calculated LOD score exceeds this threshold, there is a QTL present at $\mathrm{p}<0.05$.

For genome-wide two-dimensional scans, pairwise scans were performed at 2-cM spacing. All possible pairs of QTL locations on each chromosome were tested for association with the phenotypes. The likelihood from the full model (pseudo-marker pair and the interaction between them) and the null model (no genetic effect) was compared and LOD scores were calculated. In addition, LOD scores from comparing the likelihood from the full model and the additive model (with only the main effects of pseudo-markers and but no interaction) were calculated.

QTL and possible QTL * QTL interactions identified from a single and pair-wise QTL scans were fit into multiple regression models, and the variations of the phenotype in the models were estimated. P-values for terms in the multiple regression model were computed. Terms were dropped sequentially until all of the terms in the model were significant at the $1 \%$ level for main QTL effects and $0.1 \%$ for the interaction effects. For any QTL that was characterized as strong, effect plots were generated for the autosomal region by stratifying mice based on homozygous (BALB or $\mathrm{C} 3 \mathrm{H}$ ) or heterozygous alleles (BALB and $\mathrm{C} 3 \mathrm{H}$ ).

Genes that are both residing within the $95 \%$ confidence interval of each QTL and containing polymorphisms between $\mathrm{C} 3 \mathrm{H}$ and BALB were retrieved via biomaRt (version 2.8.1) using the NCBI mus musculus gene database (NCBIM37) and mus musculus variation database (dbSNP128). Identified SNP carrying genes were analyzed by KEGG 2 pathway analysis tool to identify potential pathways important in bone physiology, including WNT, calcium signaling, TGF- $\beta$, Notch and osteoclast differentiation.

\section{Statistics}

All data were presented as mean ( \pm standard deviation). Longitudinal changes in phenotypes were calculated as \% difference from the previous time point ( $\mathrm{n}=359$ for unloading and $\mathrm{n}=352$ for reambulation) and tested for significance with paired t-tests. Paired t-tests were also 
used to compare longitudinal (\%) changes in phenotypes during unloading vs reambulation. Normality of data distributions were tested with Gaussian curve-fitting to histograms. Coefficient of variations (COV) were used to determine the degree of dispersion in the population for a given index, and calculated as standard deviation with the mean as referent. Significance was set at $\mathrm{p}<0.05$.

\section{Results}

Baseline and longitudinal mechanical phenotypes of F2 mice

At baseline, phenotypes used to describe mechanical properties of trabecular metaphysis showed variable distributions with positive skewness values that were less than 1 for all variables (Table 1). Coefficients of variations (COV), were 38\%,12\%, 18\% and 39\%, for stiffness, MVM, PVM and SVM. Mechanical unloading induced changes of $-42 \%,-5 \%, 27 \%$ and $104 \%$ for stiffness, MVM, PVM and SVM (all $\mathrm{p}<0.01$ ). During reambulation, stiffness, MVM, PVM and SVM changed by $13 \%, 8 \%,-10 \%$ and $-22 \%$ (all $p<0.01$ ). Similar to baseline mechanical properties, longitudinal changes also showed large variations (Table 2). During unloading, COV for changes in stiffness, MVM, PVM and SVM were 46\%, 256\%, 54\% and 77\%, respectively. During reambulation, COV values were even higher, 238\%, 369\%, 78\% and 91\% for stiffness, MVM, PVM and SVM, indicating greater data variability in phenotypes compared to unloading.

\section{QTL for mechanical properties at baseline}

Genome wide associations identified significant QTLs for stiffness, MVM, PVM and SVM at baseline (Fig. 1). Multiple regression models showed that $18 \%$ of the variability for stiffness was accounted for by 3 significant QTLs. Of those 3 QTLs, one increased and two reduced apparent trabecular stiffness in the C3H strain (Fig. 2). For MVM, PVM and SVM, $15 \%, 20 \%$ and $11 \%$ of variability was explained by 3,5 , and 3 QTLs. Similar to stiffness values, these QTLs affected phenotypes of the progenitor strains differentially (Figs. 3-5). QTLs were shared on Chr1 (stiffness and PVM), Chr5 (MVM, PVM and SVM), Chr8 (stiffness, MVM and PVM), and Chr9 (stiffness and PVM), but otherwise were distinct.

\section{QTL for trabecular mechanical properties during unloading and} reambulation

Similar to baseline, several significant QTLs were associated with changes in trabecular bone's simulated mechanical properties during unloading and reambulation (Fig. 1). There were fewer significant QTLs for longitudinal changes in phenotypes than there were at baseline. No significant QTLs were detected for changes in MVM during unloading and reambulation and for SVM during unloading. For trabecular stiffness, 2 significant QTLs explained 9\% of data variability during unloading and one QTL explained $4 \%$ of data variability during reambulation. For PVM, 3 QTLs explained 14\% of data variability during unloading and one QTL explained 5\% of data variability during reambulation. Further, one QTL explained $5 \%$ of data variability in SVM during reambulation. Of those identified QTLs, one location on

Table 1

Trabecular bone mechanical properties of the distal femur at baseline.

\begin{tabular}{lllllll}
\hline & & $\mathrm{n}$ & Mean & SD & COV & Range \\
\hline \multirow{2}{*}{ wk (0) } & Stiffness [N/mm] & 466 & 2413 & 926 & $38 \%$ & 5198 \\
& MVM [MPa] & 466 & 0.49 & 0.06 & $12 \%$ & 0.36 \\
& PVM [MPa] & 466 & 1.02 & 0.19 & $18 \%$ & 1.05 \\
& SVM [MPa] & 466 & 0.52 & 0.21 & $39 \%$ & 1.14
\end{tabular}

Shown are descriptive statistics for trabecular stiffness, median Von-Mises stresses (MVM), peak Von-Mises stresses (PVM) and skewness of Von-Mises stress distributions (SVM).
Table 2

Changes in the trabecular mechanical properties of the distal femur during unloading, wk (0-3), and reambulation, wk (3-6).

\begin{tabular}{llllrrr}
\hline & & $\mathrm{n}$ & Mean & SD & \multicolumn{1}{c}{ COV } & Range \\
\hline \multirow{2}{*}{ wk (0-3) } & Stiffness [\%] & 359 & $-42 \%^{*}$ & $19 \%$ & $-46 \%$ & $109 \%$ \\
& MVM [\%] & 359 & $-5 \%^{*}$ & $14 \%$ & $-256 \%$ & $98 \%$ \\
& PVM [\%] & 359 & $27 \%^{*}$ & $15 \%$ & $54 \%$ & $101 \%$ \\
& SVM [\%] & 359 & $104 \%^{*}$ & $81 \%$ & $77 \%$ & $543 \%$ \\
wk (3-6) & Stiffness [\%] & 352 & $13 \%^{*}, * *$ & $31 \%$ & $238 \%$ & $220 \%$ \\
& MVM [\%] & 352 & $8 \% * * *$ & $31 \%$ & $369 \%$ & $397 \%$ \\
& PVM [\%] & 352 & $-10 \%^{*}, * *$ & $8 \%$ & $-78 \%$ & $61 \%$ \\
& SVM [\%] & 352 & $-22 \%^{*}, * *$ & $20 \%$ & $-91 \%$ & $124 \%$
\end{tabular}

Shown are descriptive statistics for changes in trabecular stiffness, median Von-Mises stresses (MVM), peak Von-Mises stresses (PVM) and skewness of Von-Mises stress distributions (SVM).

$* \mathrm{p}<0.01$ for longitudinal change in mean values during wk (0-3) or wk (3-6).

** $\mathrm{p}<0.05$ for longitudinal change in mean values between wk (0-3) vs wk (3-6).

Chr2 was shared between stiffness and PVM while all other QTLs were distinct from each other. The only overlap between QTLs identified at baseline and during unloading/reambulation was observed on Chr5 where PVM at baseline coincided with PVM during unloading.

\section{Correspondence of QTLs to known genes and pathways}

To identify genes of interest that reside within the 95\% confidence intervals of the identified QTL, a gene list was generated including genes that have single nucleotide polymorphisms (SNPs) between BALB and C3H strains. Based on this criterion, QTLs that were associated with trabecular stiffness had 798 genes for baseline, 1681 for unloading and 323 for reambulation. MVM had 653 genes for baseline. PVM had 1723 genes for baseline, 1610 for unloading, 208 for reambulation. SVM had 785 genes for baseline and 362 for reambulation. We considered PVM as the principal mechanical outcome variable and further analyses were performed on baseline and longitudinal changes of this phenotype to determine those genes that belonged to signaling pathways known to regulate bone formation and resorption. This list included genes such as Smad3, Bmp5 and TNF for baseline (Table 3), Bmp2, Wnt7a and Jag1 for unloading and Ptch1 for reambulation (Table 4).

\section{Discussion}

We identified specific locations within the genome that regulate trabecular bone's simulated mechanical properties both at baseline and during the removal and reintroduction of weightbearing. Across the genetically heterogeneous mouse population, the variability of the mechanical phenotypes measured longitudinally at baseline as well as during unloading and reambulation was consistent with their differential sensitivity to altered mechanical demand. At baseline, several genetic loci were associated with trabecular stiffness, peak stresses, and the quality of the stress histogram. QTLs were also identified for changes in trabecular mechanical properties during unloading and reambulation. Mechanical QTLs that were identified at baseline were mostly different from those identified during unloading or reambulation, suggesting that different sets of genes regulate trabecular mechanical properties in the young adult skeleton during homeostasis and when subjected to catabolic or anabolic mechanical signals. QTLs identified were also distinct for unloading and reambulation, further suggesting that the tissue response to alterations in mechanical demand is specific to the removal versus the application of mechanical loading related to weightbearing activities.

Progenitor strains in this study were initially selected for their differential changes in trabecular morphology during unloading [42] but trabecular bone's simulated mechanical properties had not been previously tested in the progenitor strains. Baseline effect plots analyzed in this study suggest that trabecular stiffness is greater and peak stresses are smaller in BALB mice than in C3H. They also show that, similar to trabecular bone volume fraction, peak trabecular stresses in BALB mice are 
(a)

QTL for Trabecular Stiffness at Baseline

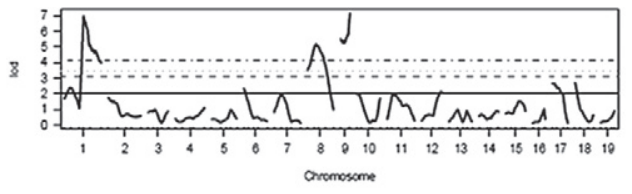

QTL for PVM at Baseline

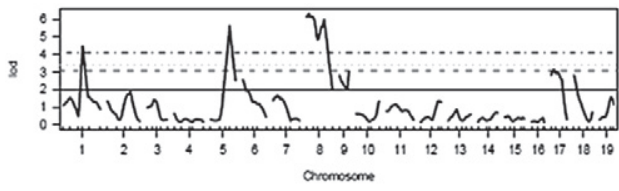

(b)

QTL for Changes in Stiffness during Unloading

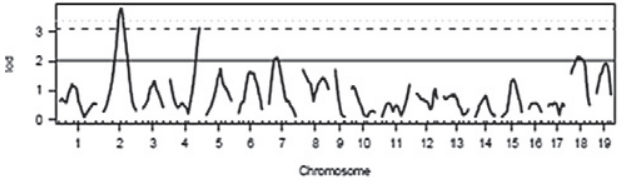

QTL for Changes in PVM during Unloading

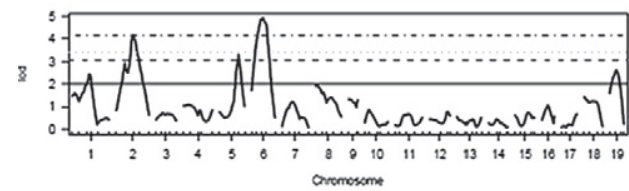

(c)

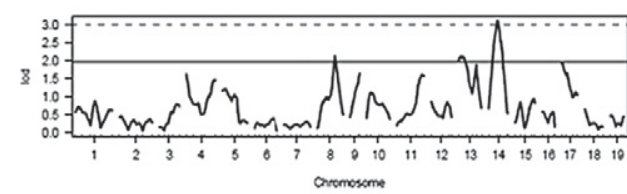

QTL for Changes in PVM during Reloading

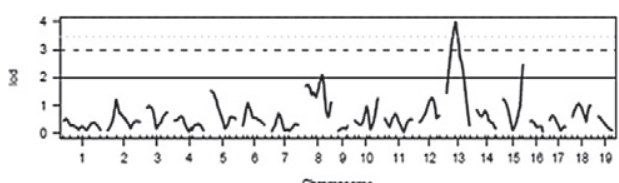

QTL for MVM at Baseline

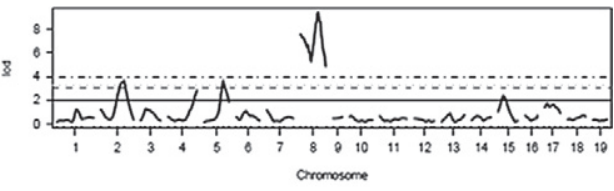

QTL for SVM at Baseline

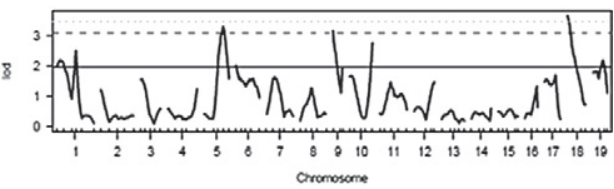

QTL for Changes in MVM during Unloading

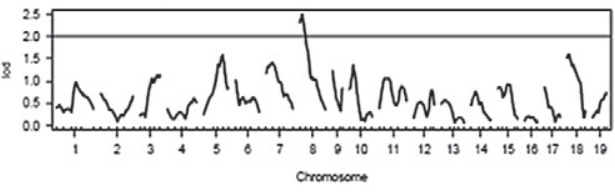

QTL for Changes in SVM during Unloading

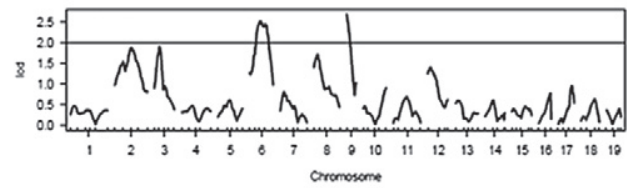

QTL for Changes in MVM during Reloading

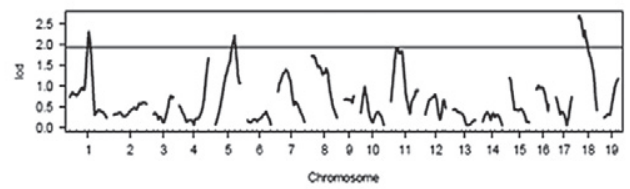

QTL for Changes in SVM during Reloading

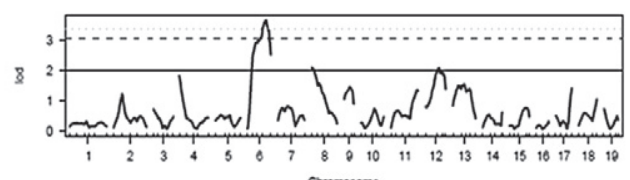

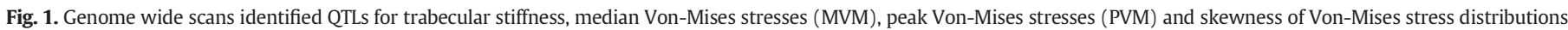

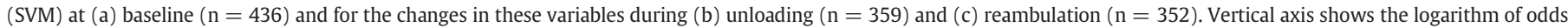

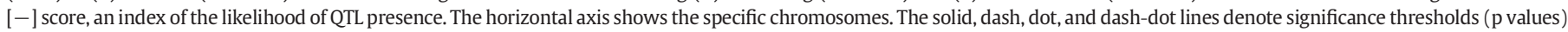
of $63 \%, 10 \%, 5 \%$, and $1 \%$, respectively. $\mathrm{p}<63 \%$ is considered suggestive for the presence of a QTL while $\mathrm{p}<1 \%$ indicates a strong likelihood of a QTL residing at this location.

more sensitive to mechanical unloading than in $\mathrm{C} 3 \mathrm{H}$. Bone's mechanical response to reambulation was also greater in BALB than in $\mathrm{C} 3 \mathrm{H}$ but the total number of QTLs for reambulation was less than for baseline or unloading. In these two mouse strains, bone's response to reambulation had not been reported previously for either morphological or mechanical properties and our results suggest some similarities between the response of $\mathrm{C} 3 \mathrm{H}$ and BALB to reconstitution of mechanical loading. Even though trabecular bone of $\mathrm{C} 3 \mathrm{H}$ is much less responsive to mechanical loading [43,44], osteoblasts of C3H mice are more active compared to many other inbred strains [45]. Perhaps, their greater basal activity levels preserved the ability of osteoblasts to respond to reloading after activity was compromised by unloading, leading to a similar reambulation response as in BALB even though BALB are known to be more responsive to mechanical loading per se [44]. While this hypothesis requires future confirmation, analyses performed here allowed us to identify QTLs for reambulation even in the presence of a milder phenotype than seen with unloading.

Morphological traits such as trabecular bone volume fraction (BV/TV), connectedness, number and thickness are all important modulators of mechanical characteristics of trabecular bone [34]. It is therefore not surprising that QTLs identified for stiffness and peak stress values at baseline overlapped with QTLs previously identified for trabecular morphology, in particular trabecular bone volume fraction [29]. Upon removal of habitual weight bearing activities, associations between morphology and mechanical properties become significantly weaker as shown in our companion article [65]. That risk of mechanical failure, the most important clinical outcome variable, is more directly associated with bone stiffness and the heterogeneity of mechanical stress distributions $[28,46]$, rather than morphology, emphasizes the importance of identifying the underlying genetic regulators. QTLs identified for the longitudinal changes in mechanical phenotypes during unloading and reambulation were not identical to those identified for BV/TV [29]. For example, QTLs for changes in BV/TV during unloading [29] were located on chromosomes 2, 3, 7, 8 , 10 and 19 while for changes in peak stresses induced by unloading, QTLs were found on chromosomes 2,5 and 6. This comparison suggests genetic regulation of the longitudinal changes in mechanical properties during unloading that is, at least in part, independent from morphologic changes in trabecular bone. 


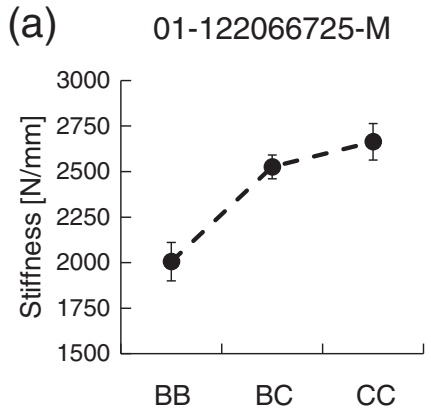

(d)

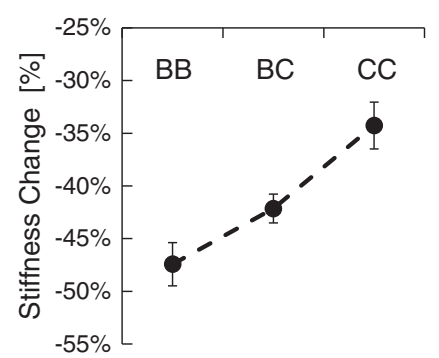

(b) 08-026008244-M

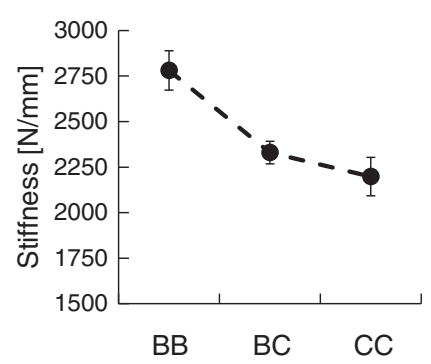

(e)

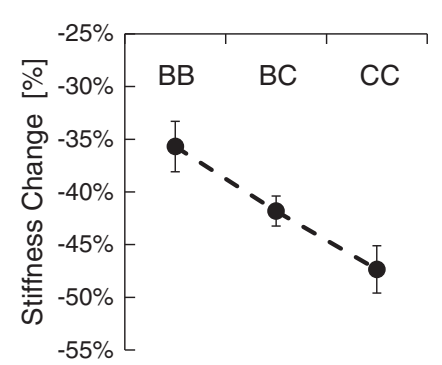

(c)

09-065820797-M

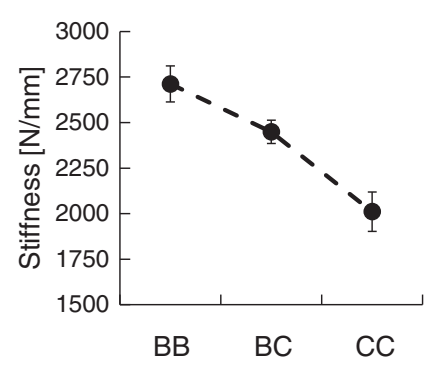

(f)

14-054014360-M

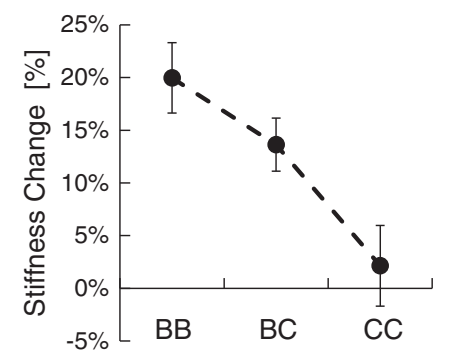

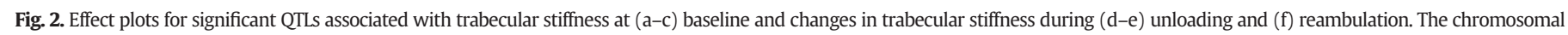

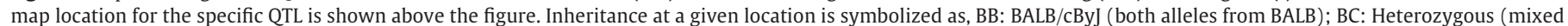
alleles); $\mathrm{CC}$ : $\mathrm{C} 3 \mathrm{H} / \mathrm{HeJ}$ (both alleles from $\mathrm{C} 3 \mathrm{H}$ ).

QTLs identified in this study partially resemble genetic loci that were previously identified as regulators of the (baseline) mechanical strength of bone. Using progenitor strains different from those in our study, QTLs pertaining to cortical breaking strength in the femur were identified by bending tests $[9,18]$. Of those QTLs, Fembrs2, Fembrs4, Fembrs6, and Bmch7 overlap with QTLs identified here for peak trabecular stress at baseline. Although these studies tested mechanical properties of cortical bone and not trabecular bone, it is entirely possible that these loci influence both cortical and trabecular mechanical properties. QTLs that identified changes in peak stresses were similar to Fembrs1 during unloading and to Bmch6 during reambulation. As these loci were previously identified in mice enjoying habitual levels of weightbearing, it appears that there are genes that modulate both mechanical properties in the young adult skeleton as well as their longitudinal changes during catabolic and anabolic mechanical signals.

Mechanical sensation and responsiveness of bone cells are facilitated by orchestrating many mechanical and chemical processes embedded within different molecular signaling pathways [47]. The genomic loci identified here house many genes and it is not entirely straightforward to determine the specific genes responsible for the trabecular mechanical traits considered here. Even when only focusing on those genes with direct relevance to pathways known to play a role in bone turnover, the list is too large to speculate on single candidate genes. Thus, QTLs identified in this study will require mechanistic follow-up studies, in vivo and/ or in vitro, for the identification of specific genes that regulate bone's mechanical properties to alterations in weight bearing. Nevertheless, it is instructive to discuss some of the genes previously associated with changes in bone formation and resorption. Of genes within the confidence intervals, a putative candidate for determining mechanical properties at baseline is bone morphogenic protein 5 (Bmp5), an important modulator of limb development and bone morphogenesis $[48,49]$ and, by extension, of mechanical strength of trabecular bone. Another example includes Smad3 which may promote osteoblastogenesis [50,51] and regulates TGF- $\beta$ induced proliferation and osteogenic commitment of mesenchymal stem cells [52]. Not surprisingly, the list of candidate genes for peak stress at baseline not only involves genes regulating bone formation but also bone resorption. For instance, tumor necrosis factor (TNF) can stimulate osteoclast recruitment [53], with strong effects on the morphology and mechanical properties of the trabecular load bearing structure. These gene targets can be validated (or refuted) in future studies that take advantage of molecular manipulation techniques such as constitutive or inducible knock-out models.

Some of the candidate genes with single nucleotide polymorphisms (SNP) between BALB and C3H that were associated with longitudinal (a)

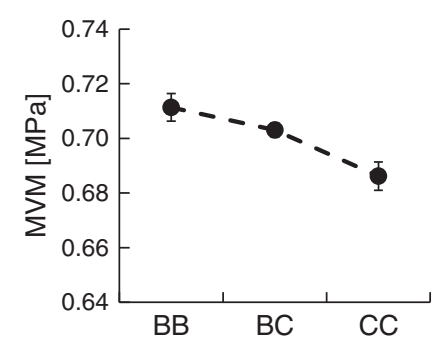

(b)

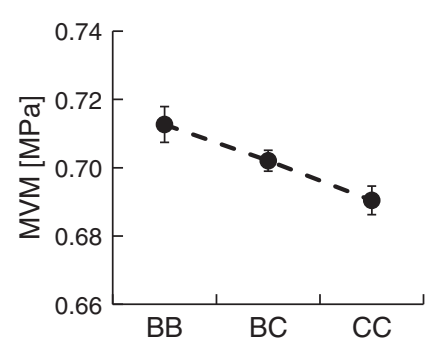

(c)

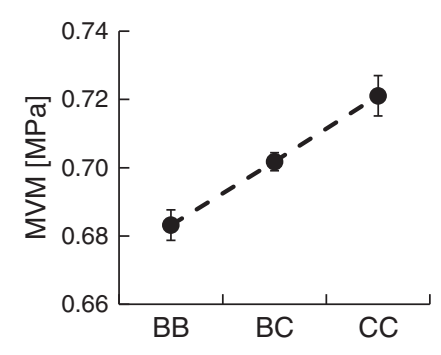

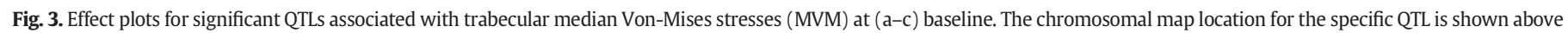
the figure. Inheritance at a given location is symbolized as, BB: BALB/cByJ (both alleles from BALB); BC: Heterozygous (mixed alleles); CC: C3H/HeJ (both alleles from C3H). 
(a) 01-122066725-M

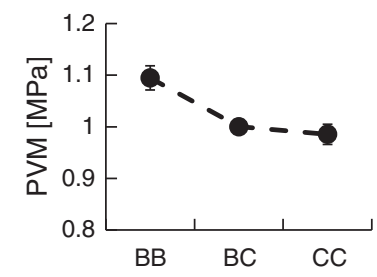

(d) 09-065820797-M

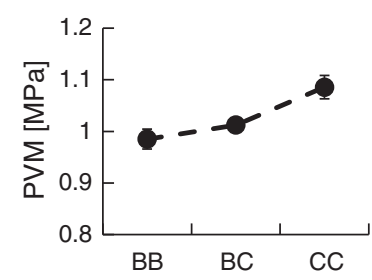

(g) $\quad 05-104525294-\mathrm{N}$

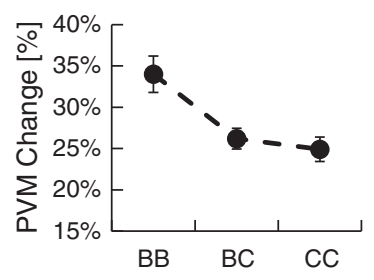

(b) $\quad 05-104525294-\mathrm{N}$

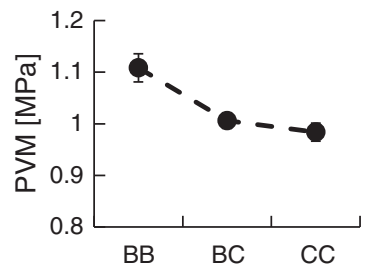

(e) 17-042164456-M

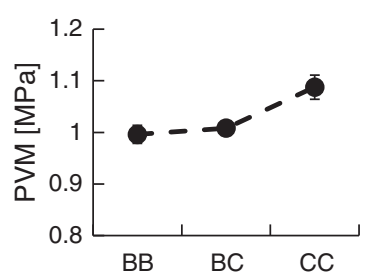

(h) $\quad 06-082087904-M$

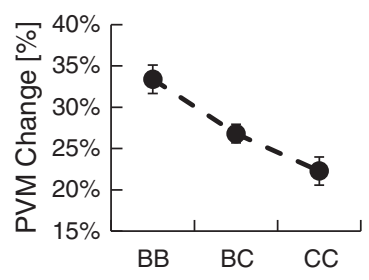

(c) $08-003089774-\mathrm{M}$

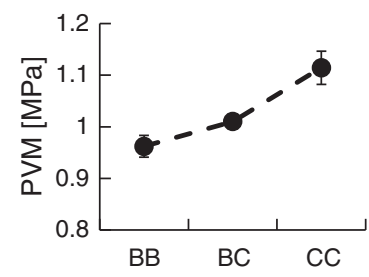

(f) 02-088002203-M

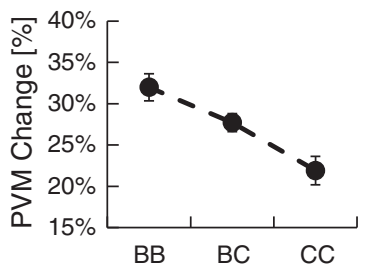

(i) $\quad 13-052612410-\mathrm{N}$

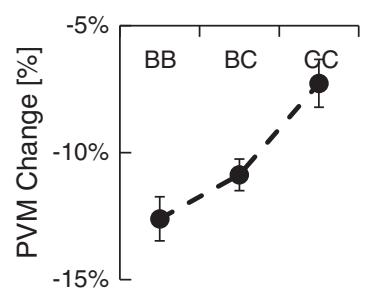

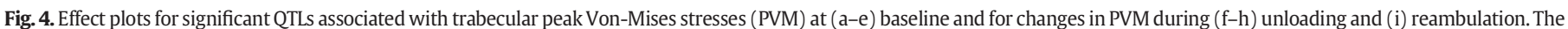

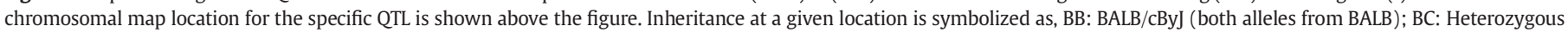
(mixed alleles); $\mathrm{CC}$ : $\mathrm{C} 3 \mathrm{H} / \mathrm{HeJ}$ (both alleles from $\mathrm{C} 3 \mathrm{H}$ ).

(a)

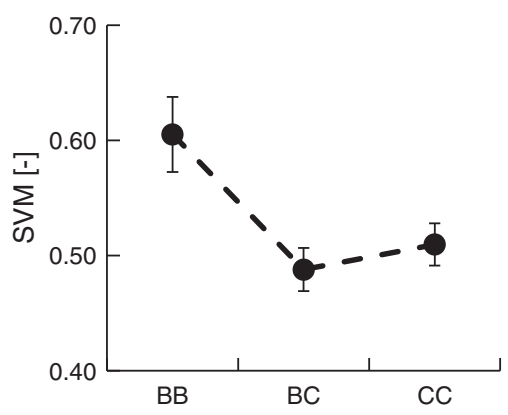

(c)

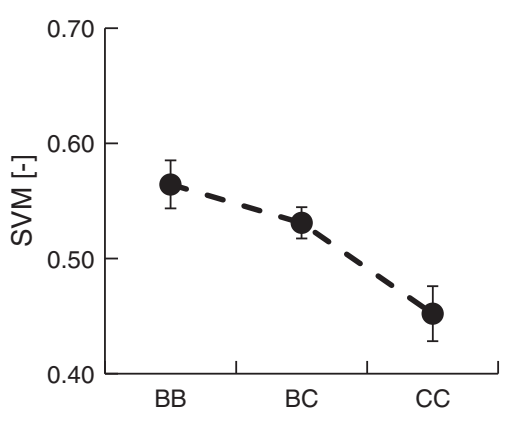

(b)

D9Mit297

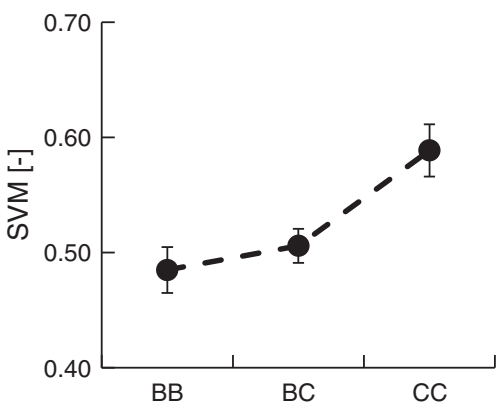

(d) 06-112964989-M

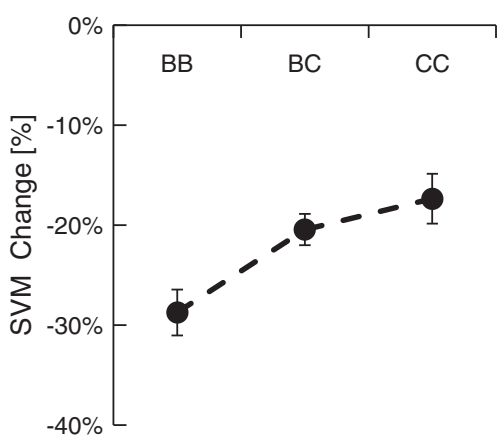

Fig. 5. Effect plots for significant QTLs associated with trabecular skewness of Von-Mises stress distributions (SVM) at (a-c) baseline and for changes in SVM during (d) unloading. The chromosomal map location for the specific QTL is shown above the figure. Inheritance at a given location is symbolized as, BB: BALB/CByJ (both alleles from BALB); BC: Heterozygous (mixed alleles); $\mathrm{CC}: \mathrm{C} 3 \mathrm{H} / \mathrm{HeJ}$ (both alleles from $\mathrm{C} 3 \mathrm{H}$ ). 
Table 3

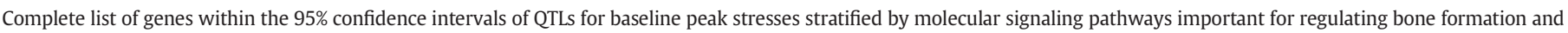
resorption.

\begin{tabular}{|c|c|}
\hline Signaling pathway & Associated genes within QTL \\
\hline WNT & Cacybp, Ccnd3, Csnk2a2, Csnk2b, Fzd10, Gm9840, Nfat5, Nkd1, Ppard, Prkaca, Siah1a, Smad3 \\
\hline Calcium & Adcy7, Adrb3, Cacna1a, Cacna1h, Cacna1s, Ednra, Itpr3, Mylk3, Orai1, P2rx4, P2rx7, Phkb, Phkg1, Prkaca \\
\hline TGF- $\beta$ & Bmp5, Comp, Gm9840, Smad3, Smad6, Thbs2, Tnf \\
\hline Osteoclast differentiation & Cyld, Map2k1, Map2k7, Mapk13, Mapk14, Tnf \\
\hline NF-kappa B & Csnk2a2, Csnk2b, Lta, Ltb, Ptgs2, Tnf \\
\hline Notch & Aph1b, Aph1c, Dll1, Kat2b, Ncor2, Notch3, Notch4 \\
\hline Hedgehog & Csnk1g1, Prkaca \\
\hline Actin cytoskeletal regulation & Arhgef7, Bcar1, Itga11, Map2k1, Mylk3 \\
\hline ECM-receptor interaction & Col11a2, Col4a2, Comp, Itga11, Lamc1, Thbs2, Tnn, Tnr, Tnxb \\
\hline Focal adhesion & Bcar1, Ccnd3, Col11a2, Col4a2, Comp, Itga11, Lamc1, Map2k1, Mylk3, Pdpk1, Thbs2, Tln2, Tnn, Tnr, Tnxb, Vegfa, Vegfc \\
\hline Gap junctional communication & Adcy7, Itpr3, Map2k1, Map2k5, Prkaca, Tubb5 \\
\hline
\end{tabular}

changes in peak stresses during unloading and reambulation are critical for physiologic processes in bone. For example, bone morphogenic protein 2 (Bmp2), a potential regulator of mechanical risk of fracture during unloading identified here is not only an important determinant of bone morphology [54] but may also interact with mechanical signals during anabolism [55]. Moreover, Bmp2 is down-regulated in bone cells during mechanical unloading [56] and up-regulated with mechanical loading [57], suggesting that Bmp2 is well integrated into the signaling cascades that modulate changes in mechanical demand. Perhaps of equal importance, Jag1 is a gene known to regulate bone mineral density, reduces susceptibility to fracture, and sensitizes bone cells for the anabolic effects of PTH hormone [58,59]. Jag- 1 is down-regulated in bone cells during mechanical loading in vitro, perhaps increasing cell proliferation [60]. Another potential regulator identified for changes in peak stress during unloading is Wnt7a, a gene with roles in the promotion of bone formation [61]. Mutations in Wnt7a can cause malformations in long bones [62]. Genes modulating the magnitude of the change in peak trabecular stress during reambulation were fewer in numbers compared to baseline or unloading because of the presence of fewer QTL. Among these genes, Ptch1 is a negative regulator of Hedgehog signaling pathway and may play a role for the development of trabecular bone [63] and the response of osteoprogenitor cells to mechanical loading [64].

While the identity of genetic determinants of trabecular bone's mechanical properties and their changes to decreased and increased mechanical demands is still emerging, this linkage study used three different conditions - baseline, unloading, and reambulation - to determine genomic locations that are associated with regulation of trabecular bone mechanical properties. While the longitudinal nature of this study precluded an invasive characterization of material properties known to modulate bone's mechanical properties, including microcrack accumulation

Table 4

Complete list of genes within the 95\% confidence intervals of QTLs for changes in peak stress during unloading and reambulation (in bold) stratified by molecular signaling pathways important for regulating bone formation and resorption.

\begin{tabular}{ll}
\hline Signaling pathway & Associated genes within QTL \\
\hline WNT & Csnk2a1, Fzd10, Plcb1, Plcb2, Prickle2, Ruvbl1, \\
& Wnt7a \\
Calcium & Adra1d, Atp2b2, Cacna1c, Chrm5, Hrh1, Itpka, \\
& Itpr1, Nos1, Orai1, P2rx4, P2rx7, Pde1a, Phkg1, \\
& Plcb1, Plcb2, Ryr3, Tacr1, Adcy2, Agtr1a, Mylk4 \\
TGF- $\beta$ & Acvr1, Acvr1c, Acvr2a, Bmp2 \\
Osteoclast differentiation & Il1a, Il1b, Mitf, Pparg, Sfpi1, Sirpa \\
NF-kappa B & Csnk2a1, Erc1, Il1b \\
Notch & Cir1, Dll4, Jag1, Ncor2 \\
Hedgehog & Bmp2, Wnt7a, Gas1, Ptch1 \\
Actin cytoskeletal regulation & Arpc4, Chrm4, Chrm5, F2, Fgf7, Itga4, Itga6, Pak6, \\
& Pak7, Pxn, Raf1, Ssh1, Mylk4, Pfn3 \\
ECM-receptor interaction & Cd44, Itga4, Itga6 \\
Focal adhesion & Itga4, Itga6, Pak6, Pak7, Pxn, Raf1, Shc4, Mylk4, \\
& Shc3 \\
Gap junctional communication & Gjd2, Itpr1, Plcb1, Plcb2, Raf1, Gas1, Ptch1 \\
\hline
\end{tabular}

and bone's chemical properties, we identified novel QTLs for changes in bone's mechanical properties simulated by FE models. These QTLs for dynamic processes partially differ from QTLs previously identified for trabecular morphology and cortical mechanical properties during homeostasis. Determining specific candidate genes within QTLs and confirming them in humans may facilitate the genetic identification of those individuals that are at greatest risk for fracture before and during unloading and those whose trabecular mechanical properties will benefit the most during reambulation.

\section{Funding source}

NASA.

\section{Conflict of interest}

Authors have no conflicts of interest.

\section{Acknowledgments}

This research was kindly funded by NASA NAG 9-1499, NNX08BA35G and NNX12AL25G. Outstanding technical support from Shiyun Xu, Svetlana Lublinsky, Andres Laib, and Bert van Rietbergen was greatly appreciated.

\section{References}

[1] Ozcivici E, Luu YK, Adler B, Qin YX, Rubin J, Judex S, et al. Mechanical signals as anabolic agents in bone. Nat Rev Rheumatol 2010;6:50-9.

[2] Robling AG, Turner $\mathrm{CH}$. Mechanical signaling for bone modeling and remodeling. Crit Rev Eukaryot Gene Expr 2009;19:319-38.

[3] Alexandre C, Vico L. Pathophysiology of bone loss in disuse osteoporosis. Joint Bone Spine 2011;78:572-6.

[4] Zhang P, Hamamura K, Yokota H. A brief review of bone adaptation to unloading. Genomics Proteomics Bioinformatics 2008;6:4-7.

[5] Ozcivici E. Effects of spaceflight on cells of bone marrow origin. Turk J Hematol 2013;30:1-7.

[6] Turner CH, Hsieh YF, Muller R, Bouxsein ML, Baylink DJ, Rosen CJ, et al. Genetic regulation of cortical and trabecular bone strength and microstructure in inbred strains of mice. J Bone Miner Res 2000;15:1126-31.

[7] Dimai HP, Linkhart TA, Linkhart SG, Donahue LR, Beamer WG, Rosen CJ, et al. Alkaline phosphatase levels and osteoprogenitor cell numbers suggest bone formation may contribute to peak bone density differences between two inbred strains of mice. Bone 1998;22:211-6.

[8] Judex S, Garman R, Squire M, Donahue LR, Rubin C. Genetically based influences on the site-specific regulation of trabecular and cortical bone morphology. J Bone Miner Res 2004:19:600-6.

[9] Volkman SK, Galecki AT, Burke DT, Miller RA, Goldstein SA. Quantitative trait loci that modulate femoral mechanical properties in a genetically heterogeneous mouse population. J Bone Miner Res 2004;19:1497-505.

[10] Bouxsein ML, Uchiyama T, Rosen CJ, Shultz KL, Donahue LR, Turner CH, et al. Mapping quantitative trait Loci for vertebral trabecular bone volume fraction and microarchitecture in mice. J Bone Miner Res 2004;19:587-99.

[11] Lang DH, Sharkey NA, Mack HA, Vogler GP, Vandenbergh DJ, Blizard DA, et al. Quantitative trait loci analysis of structural and material skeletal phenotypes in C57BL/6 J and DBA/2 second-generation and recombinant inbred mice. J Bone Miner Res 2005;20:88-99. 
[12] Bower AL, Lang DH, Vogler GP, Vandenbergh DJ, Blizard DA, Stout JT, et al. QTL analysis of trabecular bone in BXD F2 and RI mice. J Bone Miner Res 2006;21:1267-75.

[13] Jepsen KJ, Courtland HW, Nadeau JH. Genetically determined phenotype covariation networks control bone strength. J Bone Miner Res 2010;25:1581-93.

[14] Mukherjee A, Larson EA, Carlos AS, Belknap JK, Rotwein P, Klein RF. Congenic mice provide in vivo evidence for a genetic locus that modulates intrinsic transforming growth factor beta1-mediated signaling and bone acquisition. J Bone Miner Res 2012;27:1345-56.

[15] Klein OF, Carlos AS, Vartanian KA, Chambers VK, Turner EJ, Phillips TJ, et al. Confirmation and fine mapping of chromosomal regions influencing peak bone mass in mice. J Bone Miner Res 2001;16:1953-61.

[16] Cole JH, van der Meulen MC. Whole bone mechanics and bone quality. Clin Orthop Relat Res 2011;469:2139-49.

[17] Edwards WB, Schnitzer TJ, Troy KL. Torsional stiffness and strength of the proximal tibia are better predicted by finite element models than DXA or QCT. J Biomech 2013;46:1655-62.

[18] Li X, Masinde G, Gu W, Wergedal J, Mohan S, Baylink DJ. Genetic dissection of femur breaking strength in a large population (MRL/MpJ x SJL/J) of F2 Mice: single QTL effects, epistasis, and pleiotropy. Genomics 2002;79:734-40.

[19] Wergedal JE, Ackert-Bicknell CL, Tsaih SW, Sheng MH, Li R, Mohan S, et al. Femur mechanical properties in the F2 progeny of an NZB/B1NJ x RF/J cross are regulated predominantly by genetic loci that regulate bone geometry. J Bone Miner Res 2006;21:1256-66.

[20] Reeves GM, McCreadie BR, Chen S, Galecki AT, Burke DT, Miller RA, et al. Quantitative trait loci modulate vertebral morphology and mechanical properties in a population of 18-month-old genetically heterogeneous mice. Bone 2007;40:433-43.

[21] Saless N, Litscher SJ, Lopez Franco GE, Houlihan MJ, Sudhakaran S, Raheem KA, et al. Quantitative trait loci for biomechanical performance and femoral geometry in an intercross of recombinant congenic mice: restriction of the Bmd7 candidate interval. FASEB J 2009;23:2142-54.

[22] Saless N, Litscher SJ, Vanderby R, Demant P, Blank RD. Linkage mapping of principal components for femoral biomechanical performance in a reciprocal HCB- $8 \times \mathrm{HCB}-$ 23 intercross. Bone 2011:48:647-53.

[23] McCreadie BR, Goldstein SA. Biomechanics of fracture: is bone mineral density sufficient to assess risk? J Bone Miner Res 2000;15:2305-8.

[24] Klein RF. Genetic regulation of bone mineral density in mice. J Musculoskelet Neuronal Interact 2002;2:232-6.

[25] Van RB, Huiskes R, Eckstein F, Ruegsegger P. Trabecular bone tissue strains in the healthy and osteoporotic human femur. J Bone Miner Res 2003;18:1781-8.

[26] Cody DD, Gross GJ, Hou FJ, Spencer HJ, Goldstein SA, Fyhrie DP. Femoral strength is better predicted by finite element models than QCT and DXA.J Biomech 1999;32:1013-20.

[27] Crawford RP, Cann CE, Keaveny TM. Finite element models predict in vitro vertebral body compressive strength better than quantitative computed tomography. Bone 2003;33:744-50.

[28] Keaveny TM. Biomechanical computed tomography-noninvasive bone strength analysis using clinical computed tomography scans. Ann N Y Acad Sci 2010;1192:57-65.

[29] Judex S, Zhang W, Donahue LR, Ozcivici E. Genetic loci that control the loss and regain of trabecular bone during unloading and reambulation. J Bone Miner Res 2013;28:1537-49.

[30] Morey-Holton ER, Globus RK. Hindlimb unloading rodent model: technical aspects. ] Appl Physiol 2002;92:1367-77.

[31] Ozcivici E, Luu YK, Rubin CT, Judex S. Low-level vibrations retain bone marrow's osteogenic potential and augment recovery of trabecular bone during reambulation. PLoS One 2010:5:e11178.

[32] Bouxsein ML, Boyd SK, Christiansen BA, Guldberg RE, Jepsen KJ, Muller R. Guidelines for assessment of bone microstructure in rodents using micro-computed tomography. J Bone Miner Res 2010;25:1468-86.

[33] Lublinsky S, Ozcivici E, Judex S. An automated algorithm to detect the trabecularcortical bone interface in micro-computed tomographic images. Calcif Tissue Int 2007;81:285-93.

[34] Ozcivici E, Garman R, Judex S. High-frequency oscillatory motions enhance the simulated mechanical properties of non-weight bearing trabecular bone. J Biomech 2007;40:3404-11.

[35] Busa B, Miller LM, Rubin CT, Oin YX, Judex S. Rapid establishment of chemical and mechanical properties during lamellar bone formation. Calcif Tissue Int 2005;77:386-94.

[36] Rho JY, Tsui TY, Pharr GM. Elastic properties of human cortical and trabecular lamellar bone measured by nanoindentation. Biomaterials 1997;18:1325-30.

[37] Bayraktar HH, Morgan EF, Niebur GL, Morris GE, Wong EK, Keaveny TM. Comparison of the elastic and yield properties of human femoral trabecular and cortical bone tissue. J Biomech 2004;37:27-35.

[38] Broman KW, Wu H, Sen S, Churchill GA. R/qtl: QTL mapping in experimental crosses. Bioinformatics 2003;19:889-90.
[39] Sen S, Churchill GA. A statistical framework for quantitative trait mapping. Genetics 2001;159:371-87.

[40] Doerge RW, Churchill GA. Permutation tests for multiple loci affecting a quantitative character. Genetics 1996;142:285-94.

[41] Lander E, Kruglyak L. Genetic dissection of complex traits: guidelines for interpreting and reporting linkage results. Nat Genet 1995;11:241-7.

[42] Judex S, Garman R, Squire M, Busa B, Donahue LR, Rubin C. Genetically linked sitespecificity of disuse osteoporosis. J Bone Miner Res 2004;19:607-13.

[43] Kodama Y, Umemura Y, Nagasawa S, Beamer WG, Donahue LR, Rosen CR, et al Exercise and mechanical loading increase periosteal bone formation and whole bone strength in $\mathrm{C} 57 \mathrm{BL} / 6 \mathrm{~J}$ mice but not in $\mathrm{C} 3 \mathrm{H} / \mathrm{Hej}$ mice. Calcif Tissue Int 2000;66:298-306.

[44] Judex S, Donahue LR, Rubin C. Genetic predisposition to low bone mass is paralleled by an enhanced sensitivity to signals anabolic to the skeleton. FASEB J 2002;16:1280-2.

[45] Sheng MH, Lau KH, Beamer WG, Baylink DJ, Wergedal JE. In vivo and in vitro evidence that the high osteoblastic activity in $\mathrm{C} 3 \mathrm{H} / \mathrm{HeJ}$ mice compared to $\mathrm{C} 57 \mathrm{BL} / 6 \mathrm{~J}$ mice is intrinsic to bone cells. Bone 2004;35:711-9.

[46] Fields AJ, Lee GL, Liu XS, Jekir MG, Guo XE, Keaveny TM. Influence of vertical trabeculae on the compressive strength of the human vertebra. J Bone Miner Res 2011;26:263-9.

[47] Thompson WR, Rubin CT, Rubin J. Mechanical regulation of signaling pathways in bone. Gene 2012;503:179-93.

[48] Zuzarte-Luis V, Montero JA, Rodriguez-Leon J, Merino R, Rodriguez-Rey JC, Hurle JM. A new role for BMP5 during limb development acting through the synergic activation of Smad and MAPK pathways. Dev Biol 2004;272:39-52.

[49] Cho TJ, Gerstenfeld LC, Einhorn TA. Differential temporal expression of members of the transforming growth factor beta superfamily during murine fracture healing. Bone Miner Res 2002;17:513-20.

[50] Sowa H, Kaji H, Yamaguchi T, Sugimoto T, Chihara K. Smad3 promotes alkaline phosphatase activity and mineralization of osteoblastic MC3T3-E1 cells. J Bone Miner Res 2002;17:1190-9.

[51] Kaji H, Naito J, Sowa H, Sugimoto T, Chihara K. Smad3 differently affects osteoblast differentiation depending upon its differentiation stage. Horm Metab Res 2006;38:740-5.

[52] Jian H, Shen X, Liu I, Semenov M, He X, Wang XF. Smad3-dependent nuclear translocation of beta-catenin is required for TGF-beta1-induced proliferation of bone marrow-derived adult human mesenchymal stem cells. Genes Dev 2006;20:666-74.

[53] Asagiri M, Takayanagi H. The molecular understanding of osteoclast differentiation. Bone 2007; 40:251-64.

[54] Bessa PC, Casal M, Reis RL. Bone morphogenetic proteins in tissue engineering: the road from the laboratory to the clinic, part I (basic concepts). J Tissue Eng Regen Med 2008;2:1-13.

[55] Kopf J, Petersen A, Duda GN, Knaus P. BMP2 and mechanical loading cooperatively regulate immediate early signalling events in the BMP pathway. BMC Biol 2012;10:37.

[56] Dai Z, Wu F, Chen J, Xu H, Wang H, Guo F, et al. Actin microfilament mediates osteoblast $\mathrm{Cbfa} 1$ responsiveness to BMP2 under simulated microgravity. PLoS One 2013;8:e63661.

[57] Rath B, Nam J, Knobloch TJ, Lannutti JJ, Agarwal S. Compressive forces induce osteogenic gene expression in calvarial osteoblasts. J Biomech 2008;41:1095-103.

[58] Kung AW, Xiao SM, Cherny S, Li GH, Gao Y, Tso G, et al. Association of JAG1 with bone mineral density and osteoporotic fractures: a genome-wide association study and follow-up replication studies. Am J Hum Genet 2010;86:229-39.

[59] Weber JM, Forsythe SR, Christianson CA, Frisch BJ, Gigliotti BJ, Jordan CT, et al. Parathyroid hormone stimulates expression of the Notch ligand Jagged1 in osteoblastic cells. Bone 2006;39:485-93.

[60] Kurpinski K, Chu J, Hashi C, Li S. Anisotropic mechanosensing by mesenchymal stem cells. Proc Natl Acad Sci U S A 2006;103:16095-100.

[61] Cheng SL, Shao JS, Cai J, Sierra OL, Towler DA. Msx2 exerts bone anabolism via canonical Wnt signaling. J Biol Chem 2008;283:20505-22.

[62] Kantaputra PN, Mundlos S, Sripathomsawat W. A novel homozygous Arg222Trp missense mutation in WNT7A in two sisters with severe Al-Awadi/Raas-Rothschild/Schinzel phocomelia syndrome. Am J Med Genet A 2010;152A:2832-7.

[63] Mak KK, Kronenberg HM, Chuang PT, Mackem S, Yang Y. Indian hedgehog signals independently of PTHrP to promote chondrocyte hypertrophy. Development 2008;135:1947-56.

[64] Hoey DA, Tormey S, Ramcharan S, O'Brien FJ, Jacobs CR. Primary ciliamediated mechanotransduction in human mesenchymal stem cells. Stem Cells 2012;30:2561-70

[65] Ozcivici E, Judex S. Trabecular Bone Recovers from Mechanical Unloading Primarily by Restoring its Mechanical Function rather than its Morphology. Bone 2014. http://dx.doi.org/10.1016/j.bone.2014.05.009 In Press. 